\title{
LES DERMATHOPHYTES A PARIS DE SABOURAUD A NOS JOURS
}

\author{
G. BADILLET \\ Laboratoire Alibert, Clinique dermatologique du Pr. Puissant. \\ Hopital Saint-Louis, París. France.
}

\section{RESUMEN}

Se efectúa una revisión de los dermatófitos aislados en Paris desde la época de Sabouraud a nuestros dias, demostrándose los profundos cambios detectados desde el fin del siglo pasado. Los parásitos más activos como Trichophyton tonsurans y Microsporum audouinii, han casi desaparecido. Trichophyton rubrum, sin embargo, se ha propagado en forma fulgurante después del fin de la segunda guerra mundial, transformándose en el más frecuente parásito füngico de la población de Paris, sobrepasando ampliamente a Trichophyton interdigitale, Epidermophyton floccosum y Microsporum canis.

La diversificación de especies se debe principalmente a la importante inmigración y a los paseos dominicales y vacionales del parisino a las zonas rurales.

- Se comenta la presencia de los diversos agentes füngico: sus variaciones y porcentajes según las caracteristicas clinicas de las lesiones.

\section{SUMMARY}

$A$ revision is made of dermatophytes in Paris from the time of Sabouraud to the present, demoustrating the deep changes detected since the end of last century. The most active parasites as Trichophyton tonsurans and Microsporum audouinii, have almost disappeared. Nevertheless, Trichophyton rubrum propagated very strongly after the end of the Second World War, becoming the most frequent parasite of the population of Paris, overpassing largely Trichophyton interdigitale, Epidermophyton floccosum and Microsporum canis. The diversification of species is due mostly to the important amount of inmigration and to the weekend and vacational trips of parisians to rural areas.

The presence of the different fungic species is commented, and their variations and percentages according to the clinical characteristics of the lesions.
La seule statistique de Sabouraud a été incluse dans son ouvrage majeur "Les Teignes" publié en 1910. Elle portait sur 500 souches de dermatophytes récoltées de 1906 a 1908 et concernait surtout les agents de teignes de l'enfant; un seul agent d'épidermophytie, Epidermophyton floccosum, était alors connu et, s'il est bien décrit par Sabouraud, il ne figure pas dans sa statistique.

Le problème essentiel était alors effectivement celui des teignes du cuir chevelu de l'enfant dont on dépistait plus de 650 cas par an vers l'an 1900. Microsporum audouinii et Trichophyton tonsurans* étaient le plus souvent isolés; mais dans des proportions ne cessant de varier:

-en 1892-94 on rencontrait selon Sabouraud deux tors plus de teignes microsporiques que de teignes trichophytiques.

- en 1906-08 (statistique publiée en 1910) les proportions étaient inversées:

- $40 \%$ de micorspories 147 Microsporum audouinii 14 Microsporum canis.

- $60 \%$ de trichophyties 195 Trichophyton tonsurans 39 Trichophyton violaceum.

*On a regroupé ici sous des appellations actualisées les multiples noms d'espèces de Sabouraud qui ne sont plus reconnues valables.
Les principaux autres parasites cités étaient:

Trichophyton mentagrophytes

Trichophytons taviformes

Trichophyton schoenleinii

30 cas,

9 cas,

En résume

52 cas.

- Les agents de teignes tondantes microsporiques et trichophytiques représentaient $\ldots \ldots \ldots \ldots$ ............. 81\% des dermatophytes - Les agents de teignes inflammatoires .......... $\ldots \ldots \ldots \ldots \ldots$. . . . . $8 \%$ des dermatophytes - L'agent du favus ...... 11\%des dermatophytes L'application d'un traitement efficace (radiothérapie), l'éviction scolaire et l'hospitalisation à l'Ecole Lailler de l'Hôpital Saint Louis amènent progressivement une diminution du nombre des cas de teignes. De rares statistiques permettent de suivre l'évolution ultérieure de l'endémie teigneuse à Paris:

- Pignot, Rabut et Rivalier collectent de 1930 à 1937160 cas par an.

- Degos, Rabut et Rivalier citent de 1945 à 1947 , immédiatement après la fin de la 2 e guerre mondiale, 260 cas par an, puis de 1948 à 1954,170 cas par an. - Bismuth, dans sa thèse, cite entre 1967 et 1976,90 cas par an, mais les derniers chiffres qui correspondent seulement au recrutement du service du "cuir chevelu' de l'Hôpital Saint-Louis son inférieurs à la réalité. 
Le tableau suivant donne les pourcentages approximatifs indiqués par les diverses statistiques déjà citées.

TABLEAU No I

\begin{tabular}{|l|r|r|r|r|}
\hline \multicolumn{1}{|c|}{ Agents } & $\begin{array}{c}\text { Sabou- } \\
\text { raud } \\
\text { fongiques } \\
1906-08\end{array}$ & $\begin{array}{r}\text { Pignot } \\
\text { et Coll. } \\
1930-37\end{array}$ & $\begin{array}{r}\text { Degos } \\
\text { et Coll. } \\
1945-54\end{array}$ & Bismuth \\
\hline Microsporum & $33 \%$ & $51 \%$ & $52,2 \%$ & $76,5 \%$ \\
$\begin{array}{l}\text { Trichophytons } \\
\text { endothrix }\end{array}$ & $48 \%$ & $29 \%$ & $18,3 \%$ & $18,1 \%$ \\
$\begin{array}{l}\text { Microides } \\
\text { et Mégaspores }\end{array}$ & $8 \%$ & $2,4 \%$ & $7,7 \%$ & $1,7 \%$ \\
Favus & $11 \%$ & $17,6 \%$ & $21,8 \%$ & $3,6 \%$ \\
\hline
\end{tabular}

Ce tableau montre donc une augmentation constante des microspories depuis 1908 , au détriment des agents de teignes endothrix et faviques. Mais ceci correspond en fait à la progression de Microsporum canis, car Microsporum audouinii disparait progressivement au point de ne plus être jamais retrouvé depuis 1967. Trichophyton tonsurans suit exactement la même courbe descendante que Microsporum audouinii et disparait lui aussi à la même époque. Enfin les cas de favus autochtone deviennent de plus en plus rares.

Le second point sur lequel il faut insister est l'influence des immigrants, essentiellement depuis la guerre de 39-45 avec l'entrée en Frances des Nord-Africains porteurs de teignes à Trichophyton violaceum et à Trichophyton schoenleinii, des Africains noirs porteurs de teignes à Microsporum langeronii et Trichophyton soudanense et de diverses autres ethnies moins parasitées telles que Espagnols, Portugais, Yougoslaves, etc.

Mais le phénomène majeur de l'après-guerre 39-45 n'apparait pas sur les travaux déjà cités: c'est l'extraordinaire progression des agents d'épidermophyties et principalement de Trichophyton rubrum, parasite à peu près inconnu en France avant 1939 et qui représente, à lui seul maintenant, à peu près $60 \%$ du total de nos souches.

Depuis mai 1956, date de notre arrivée au Laboratoire Alibert de hopital Saint-Louis, nous nous sommes attachés à dresser année par année la liste des dermatophytes isolés. Une vue d'ensemble est maintenant possible sur 25 ans. Le tableau $\mathrm{N}^{\circ}$ II montre la progression du nombre des souches isolées; 36 cas la première année, pour dépasser 1.300 cas dans les années 1974-75. Depuis, les chiffres oscillent entre 1.200 et 1.500 par an.

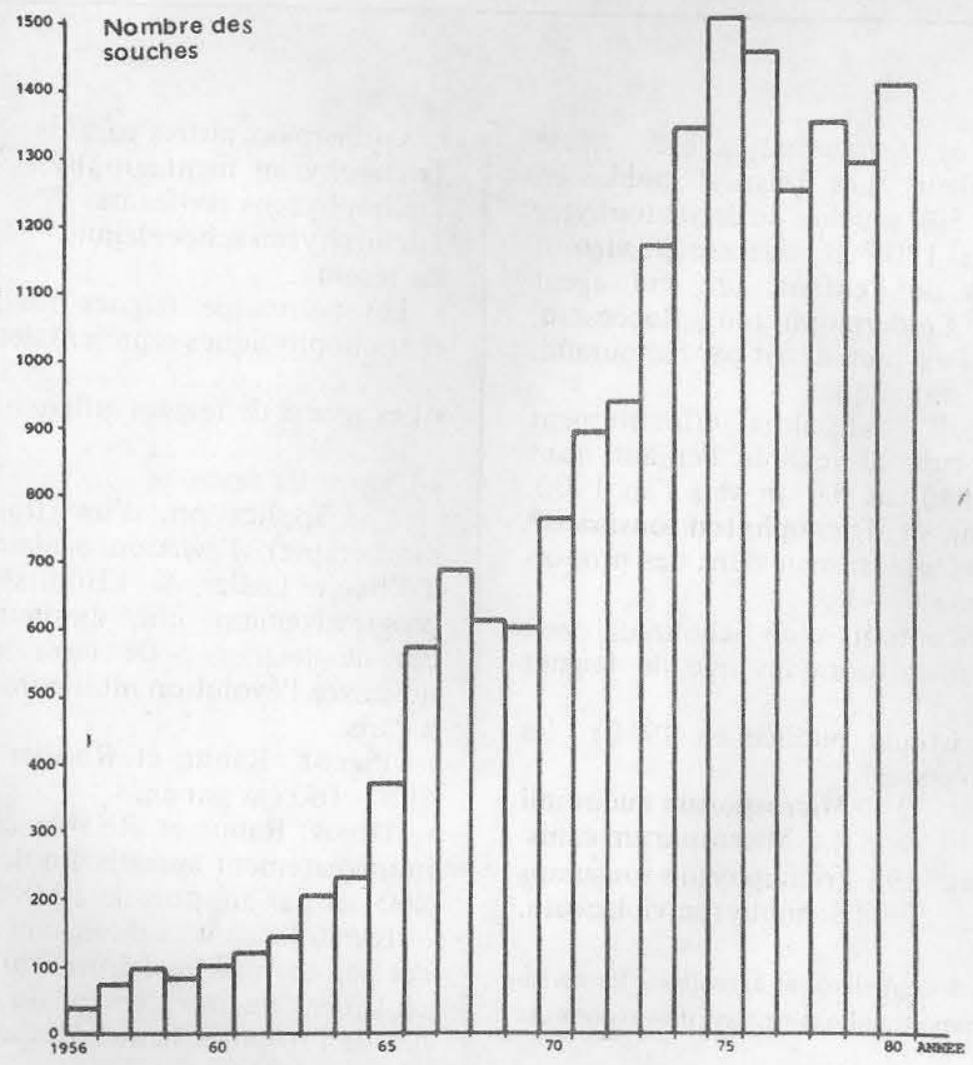


Le Tableau NO III donne l'identité des dermatophytes isolés. Ces chiffres sont regroupés par périodes de 5 ans pour faciliter leur interprétation.

Par ordre de fréquence on note donc: 9.525 souches de Trichophyton rubrum 3.282 souches de Trichophyton interdigitale 2.057 souches de Microsporum canis 1.245 souches d'Epidermophyton floccosum

16.109 au total

$=94,1 \%$

Ces 4 dermatophytes représentent donc à eux-seuls $94,1 \%$ de l'ensemble des dermatophytes.

et les agents d'épidermophyties (Trichophyton rubrum, Trichophyton interdigitale et Epidermophy. ton floccosum) $82,1 \%$ de l'ensemble des dermatophytes

Cette prédominance des 3 agents d'épidermophyties est apparue peu à peu. Par période de 5 ans, elle passe de $38 \%$ à presque $85 \%$

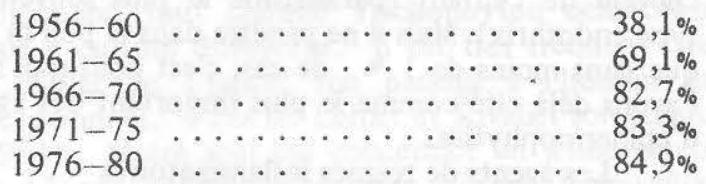

Mais si la progression étonnante de Trichophyton rubrum, qui passe de $20 \%$ à $59,4 \%$, se poursuit encore actuellement, celle de Trichophyton interdigitale est plus modeste, $12,3 \%$ à $20,6 \%$, alors qu'Epidermophyton floccosum après être passé de $5,7 \%$ à presque $15 \%$ est revenu maintenant à $5 \%$.

Le fait essentiel est donc cet envahissement par Trichophyton rubrum qui existe un peu partout en Europe mais semble atteindre en France essentiellement les citadins; les villes de province montrant un certain retard sur Paris. De nombreux cas d'eczema marginé de Hebra inguino-cruraux, bien connus des dermatologues échappent au laboratoire de Mycologie et faussent donc nos chiffres mais les lésions interdigitoplantaires et surtout les onyxis de diagnostic étiologique plus difficile nous sont par contre adressés systématiquement.

Pour ces 3 dermatophytes les lésions touchant l'enfant de 0 à 16 ans ont évolué à peu près parallèlement à celles de l'adulte. Pour Trichophyton rubrum et Trichophyton interdigitale le pourcentage des enfants est presque le même, \pm 5 \% pour Trichophyton rubrum, $\pm 4,6 \%$ pour Trichophyton interdigitale. Il est bien plus élevé pour Epidermophyton floccosum qui atteint dans plus de $10 \%$ des cas l'enfant.

\section{TABLEAU No III}

\section{EVOLUTION SUR 25 ANS DES DIFFERENTES ESPECES DE DERMATOPHYTES}

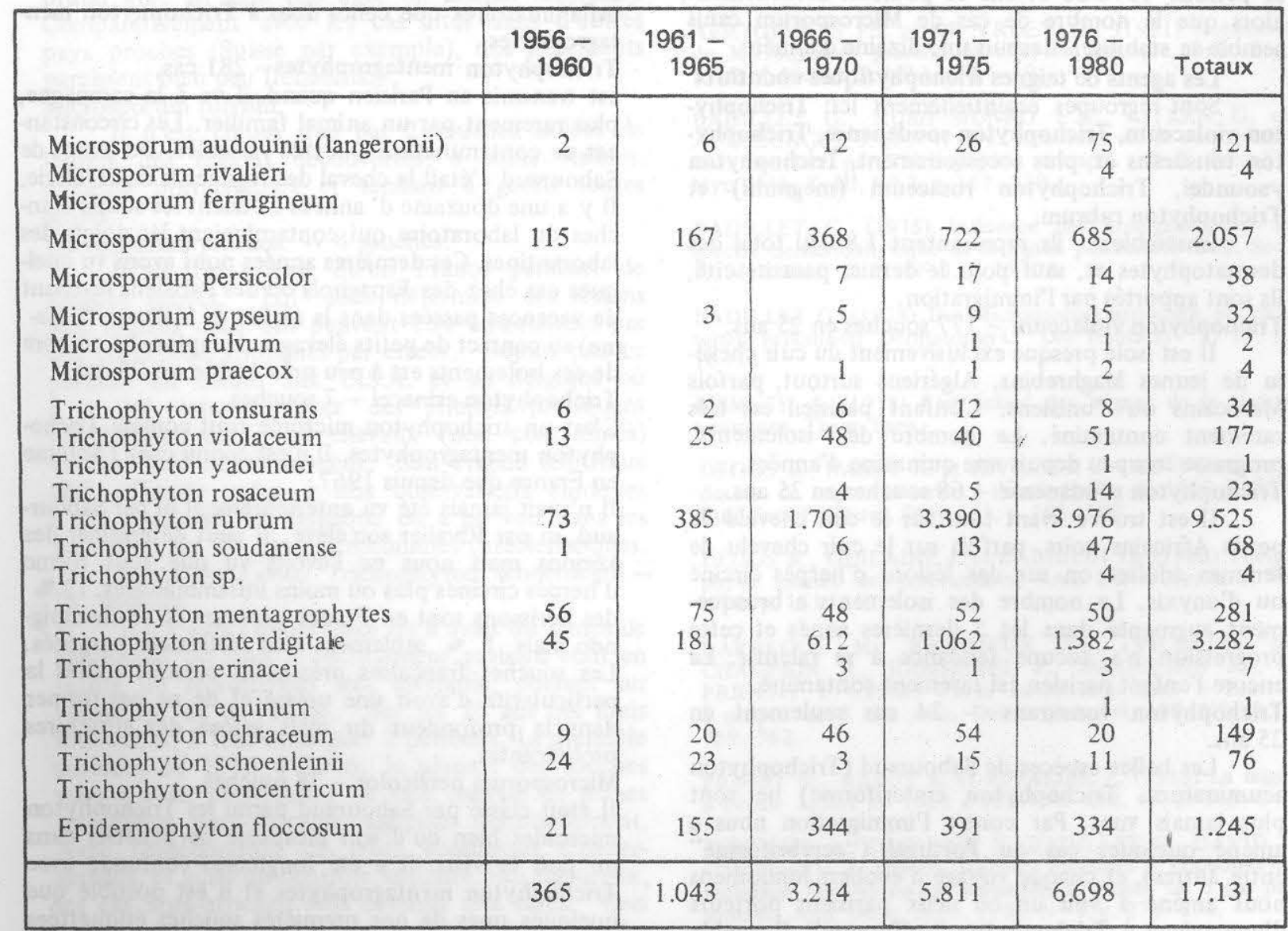


Les agents de teignes microsporiques

3 dermatophytes ont été isolés.

Microsporum canis domine largement avec 2.057 isolements:. Microsporum audouinii (ou langeronii) vient ensuite avec 121. Enfin Microsporum rivalieri n'a été vu que 4 fois.

2 types de population correspondent à ces 3 dermatophytes:

d'une part le Parisien au contact du chat, du chien ou d'autres animaux familiers, qui est parasité par Microsporum canis (les enfants présentent des teignes du cuir chevelu et des herpès circinés, les adultes presque exclusivement des herpès circinés),

d'autres part les immigrants, essentiellement des petits Africains noirs présentant des teignes du cuir chevelu à Microsporum langeronii ou Microsporum rivalieri.

On a vu également quelques cas de Parisiens contaminés par Microsporum langeronii lors d'un séjour prolongé en Afrique noire.

L'immigrant est très peu parasité par Microsporum canis. Nous en avons vu seulement quelques cas chez des Antillais et chez de jeunes Nord-Africains.

Microsporum audouinii autochtone, celui que voyait Sabouraud, a disparu de France depuis longtemps; et sous l'appellation de Microsporum audouinii, nous entendons en fait le parasite d'Afrique noire Microsporum langeronii. La progression des cas de Microsporum langeronii est très notable pour la période $1976-80$ et elle se poursuit actuellement alors que le nombre de cas de Microsporum canis semble se stabiliser despuis une dizaine d'années.

Les agents de teignes trichophytiques endothrix

Sont regroupés essentiellement ici: Trichophyton violaceum, Trichophyton soudanense, Trichophyton tonsurans et, plus accessoirement, Trichophyton yaoundei, Trichophyton rosaceum (megninii) et Trichophyton rubrum.

Ensembles*. ils représentent $1,8 \%$ du total des dermatophytes et, sauf pour le dernier parasite cité, ils sont apportés par l'immigration.

Trichophy ton violaceum - 177 souches en 25 ans.

Il est isolé presque exclusivement du cuir chevelu de jeunes Maghrebins, Algériens surtout, parfois Marocains ou Tunisiens. L'enfant parisien est très rarement contaminé. Le nombre des isolements: progresse très peu depuis une quinzaine d'années.

Trichophyton soudanense -68 souches en 25 ans.

Il est trouvé avant tout sur le cuir chevelu de petits Africains noirs, parfois sur le cuir chevelu de femmes adultes on sur des lésions d'herpès circiné ou d'onyxis. Le nombre des isolements a brusquement augmenté dans les 5 dernières annés et cette progression n'a aucune tendance à se ralentir. Là encore l'enfant parisien est rarement contaminé.

Trichophyton tonsurans - 34 cas seulement en 25 ans.

Les belles espèces de Sabouraud (Trichophyton acuminatum, Trichophyton crateriforme) ne sont plus jamais vues. Par contre l'immigration nous a amené quelques cas du Portugal ("cerebriforme" entre autres), et chaque voyage d'écoliers londoniens nous amène à voir un ou deux parisiens porteurs d'une teigne à Trichophyton "sulfureum". Il semble en effet que le passage de ces teignes à Trichophyton tonsurans variété sulfureum se fasse assez facilement de cuir chevelu d'enfant londonien à cuir chevelu d'enfant parisien.

Trichophyton rosaceum (megninii) - 23 cas en 25 ans.

Lui aussi bien que décrit par Sabouraud, avait disparu en France depuis une cinquantaine d'années.

On l'a revu vers les années 1965-70 avec les immigrés portugais, parasités au niveau de la barbe, puis sur des lésions d'herpès circiné chez les femmes portugaises, surtout aux jambes. A notre connaissance, un seul Parisien, maçon travaillant avec des Portugais, a présenté un herpès circiné de l'avant-bras.

Trichophyton yaoundei 1 seul cas,

a été isolé du cuir chevelu d'un petit Africain noir. Trichophyton rubrum doit être cité ici pour un certain nombre de cas de teignes inflammatoires de la barbe ou de la moustache, et un cas de teigne du cuir chevelu de l'enfant (parasitisme le plus souvent de type endothrix). Mais il ne pénètre dans le poil in vivo que dans moins de $1 \%$ de cas; c'est pourquoi nous l'avons déjà cité comme le plus important des agents d'épidermophyties.

Les agents de teignes inflammatoires

Il s'agit essentiellement de Trichophyton mentagrophytes et de Trichophyton ochraceum. Mais nous savons combien est arbitraire notre plan d'exposé, sachant que Trichophyton violaceum, Trichophyton soudanense, Trichophyton tonsurans et bien d'autres peuvent déterminer chez l'adulte des teignes aussi inflammatoires que celles dues à Trichophyton mentagrophytes.

Trichophyton mentagrophytes -281 cas,

est transmis au Parisien quand il va à la campagne, plus rarement par un animal familier. Les circonstances de contamination sont très variables. Du temps de Sabouraud c'était le cheval des régiments de cavalerie. Il y a une douzaine d'années c'étaient les souris blanches du laboratoire qui contaminaient les doigts des laborantines. Ces dernières années nous avons vu quelques cas chez des Espagnols ou des Parisiens revenant de vacances passées dans la région de Valence (Espagne) au contact de petits élevages de lapins. Le nombre de ces isolements est à peu près stable sur 25 ans.

Trichophyton erinacei -7 souches.

C'est un trichophyton microide tout comme Trichophyton mentagrophytes. Il n'eśt connu chez l'homme en France que depuis 1967.

Il n'avait jamais été vu antérieurement ni par Sabour. aud, ni par Rivalier son élève. Il peut déterminer des kérions mais nous ne l'avons vu que sous forme d'herpès circinés plus ou moins inflammatoires. $12 \%$ des hérissons sont en France porteurs de ce champig. non mais $1 \%$ seulement sont réellement parasités. Les souches françaises présentent presque toutes la particularité d'avoir une uréase el de ne pas former dans la profondeur du malt gélosé des structures proliférantes.

Microsporum persicolor - 38 souches,

Il était classé par Sabouraud parmi les Trichophyton microides bien qu'il soit incapable de pénétrer dans un poil in vivo. Il a été longtemps confondu avec Trichophyton mentagrophytes et il est possible que quelques unes de nos premières souches étiquettées 
Trichophyton mentagrophytes aient été en réalité Microsporum persicolor. Il détermine des herpès circinés des régions découvertes chez les Parisiens qui vont à la campagne et qui sont en contact direct ou indirect, par l'intermédiaire d'un chat, avec des petites rongeurs sauvages (campagnols, lérots, etc. ...). Le nombre des isolements ne tend pas à augmenter.

Trichophyton ochraceum ( $=$ Trichophyton verucosum) - 149 souches. Il contamine le Parisien qui va au contact des bovidés dans les fermes. Le chiffre de nos isolements tendrait plutôt à diminuer alors que c'est probablement le dermatophyte le plus actif en France si l'on écarte Trichophyton rubrum. De très nombreux élevages de bovidés sont en effet contaminés et l'entourage humain est souvent atteint; mais le diagnostic en est facile et le malade ne vient plus consulter à Paris.

Trichophyton equinum - 1 souche.

C'etait pour Sabouraud un Trichophyton. mégaspore, tout comme Trichophyton ochraceum, mais à culture duveteuse. Il est très fréquent chez le cheval en France; son passage à l'homme est exceptionnel. 4 cas de Lefort et Rivalier son connus (1954). Notre seul cas concernait un jeune garçon de 17 ans contaminé sur les cuisses; il travaillait depuis quelques semaines dans une écurie de courses.

Il reste à citer 3 parasites telluriques:

Microsporum gypseum - 32 souches.

II peut determiner des kérions du cuir chevelu ou des herpés circines des régions du corps en contact brutal avec la terre (ou avec un animal teigneux). Comparativement avec les cas cités dans d'autres pays proches (Suisse par exemple), nos isolements paraissent bien peu fréquents.

Microsporum fulvum

Il n'est connu que par 2 souches isolées de lésions des régions découvertes, à peine visibles, à peine prurigineuses et tendant à guérir toutes seules.

Microsporum praecox -4 souches.

Il ne fut connu qu'en France pendant de longues années. Lui aussi détermine des lésions très fugaces mais qui peuvent être entretenues par des corticoides appliqués par erreur. Depuis peu ce parasite est connu aux U.S.A. et en Belgique oú il a été retrouvé chez des femmes pratiquant l'équitation; chez des chevaux (non contaminés) et dans leur environnement. Son origine tellurique déjà affirmée grâce à nos observations cliniques a été confirmée en Belgique où a pu voir dans les poussières du sol des macroconidies caractéristiques.

Agent du Favus: Trichophyton schoenleinii 76 souches

Il n'a plús l'importance qu'il avait du temps de Sabouraud où tout le recrutement parisien était en réalité un recrutement très diversifié, portant sur l'ensemble de la France. Sur les 25 ans qui nous intéressent, il faut distinguer 2 périodes. La première période, de 1956 à 1966: la plupart des souches vues à Paris provenaient du cuir chevelu de jeunes travailleurs nord-africains, algériens essentiellement. Avec l'amélioration de leurs conditions d'hébergement, et aussi un controle sanitaire plus rigoureux, les cas nord-africains sont devenus très rares. Ceci correspond d'ailleurs à la raréfaction du favus dans les villes d'Algérie.
Dans la seconde période, 1966-1980, c'est essentiellement la découverte de plusieurs foyers de favus autochtones, en Bretagne et surtout en Normandie, qui nous a fourni de nouvelles souches. Le favus autochtone existe donc toujours en France et ces toutes dernières années, des cas ont été signalés dans le Poitou. Il est logique de penser que dans les années ultérieures, Trichophyton schoenleinii deviendra une espéce très rarement isolée à Paris.

\section{CONCLUSION}

Cette rapide revue des dermatophy tes à Paris nous a montré que deschangements profonds son intervenus depuis la fin du siècle dernier: les parasites les plus actifs (Trichophyton tonsurans, Microsporum audouinii) ont à peu près disparu. Trichophyton rubrum s'est par contre multiplié de façon fulgurante depuis la fin de la 2 e guerre mondiale. Devenu le parasite fongique majeur du Parisien, il n'est concurrencé que par Trichophyton interdigitale, Epidermophyton floccosum et Microsporum canis. Et si le Parisien n'allait pas à la campagne le dimanche ou pendant ses vacances, nos isolements se limiteraient pratiquement à ces 4 dermatophytes.

L'immigration, représente elle aussi une bonne source de diversification.

\section{BIBLIOGRAPHIE}

AERTGEERTS Ph, et DE VROEY Ch. (1981) Isolement de Microsporum praecox Rivalier en Belgique. Bull. Soc. Fr. Myc. Med. T X, No 1, p. 17-19.

BADILLET G., RUSH-MUNRO F.M., AITKEN G. et AUGSBURGER' J. (1974) Deux nouveaux cas de contamination humaine par Trichophyton erinacei. Bull. Soc. Fr. Myc. Med. T. III, No 2. p. 167-170.

BADILLET G. (1975) Influence des immigrants sur le spectre dermatophytique de la région parisienne. Bull. Soc. Fr. Myc. Med. T. IV, No 1 , p. $75-78$.

BADILLET G. (1978) Das Microsporum persicolor, ein nicht selten verkannter Dermatophyt. Der Hautarzt T. 29, p. 10-14.

BISMUTH S. (1978) Etat actuel des teignes de la région parisienne. Thèse, Paris.

DEGOS R., RABUT R. et RIVALIER E. (1954) Statistique des cas de teignes observés à l'école Lailler de 1950 à 1953. Bull. Soc. Fr. Derm. Syph. 61, p. 108-109.

DEGOS R., GUILAINE J, et BADILLET G. (1968) Trichophytie murine des laborantines. Ann. Derm. Syph. T. 95, No 2, p. $161-165$.

LARREGUE M., BRESSIEUX J.M., VILLATTE D., CHAMPION R., RAMDENEE P., JACQUEMIN J.L. et PRIGENT F. (1981) Favus autochtone dans le Poitou: à propos de 3 cas. Ann. Derm. Ven, vol. 108, No 10, p. $759-762$.

PIGNOT M., RABUT R. et RIVALIER E. (1938) La teigne à l'école Lailler de 1930 à 1937. Pr. Med. 46, p. 345-347.

PUISSANT A., DROUHET E., BADILLET G., DAVID V. et MALARD A. (1969). Mycoses étendues à Trichophyton rosaceum (Trichophyton megninii) à propos de 3 cas personnels. Ann. Derm. Syph. T. 96, No 3, p. 271-278.

SABOURAUD R. (1910) Les Teignes. Masson. 